\title{
NOTE ON GALOIS COHOMOLOGY
}

\author{
TADASI NAKAYAMA
}

Let $K_{/}^{\prime} k$ be a Galois extension. Formerly the writer studied, in [3], [4], a certain correlation of factor sets in $K / k$ with the norm class group of $K / k$, and extended it, in $[\bar{j}]$, to 3 -dimensional cocycles. The present note is to study the same relationship for general $n$-cocycles. As a matter of fact, the constructions which underlie the relationship have become common places in cohomology theory, through the works of French and American authors, and indeed the construction to bring certain (non-Galois) cocycles into the ground field $k$ has been discussed by Baer [1, Theorem C] for general dimensions $n$ under the setting of general group cohomology. Since, however, to assume the triviality of the 2- (and higher) cohomology groups is too strong (and rather meaningless) in the Galois cohomology case, ${ }^{1)}$ what we may do is, firstly, to assume certain $(n-2)-,(n$ $-3)$-, . . 2-cocycles appearing successively in the reduction, rather than the corresponding whole cohomology groups, to be $\sim 1$, as was briefly indicated in [5], or, secondly, to construct, in an also rather well-known way, 2- or 3-cocycles from the original given $n$-cocycles and apply our former results to them. For an even $n$ these are about all we shall discuss in the following. But, for an odd $n$ there is a certain construction which is similar to the latter of the above constructions but is more general. The writer assumes that this last is somehow worth while to report (Theorem 2); even the above constructions do not seem to the writer to be contained in what has been explicitly stated formerly, in [1] for instance." We shall also extend our former 3-dimensional generalization ${ }^{31}$ of the Witt-Akizuki formula to higher dimensions; such possibility being rather expected. The note is a preliminary for a succeeding one in which the writer wants to study the obtained correlations in arithmetical case.

1. Preliminary observations. Let $K / k$ be a Galois extension and $G$ be its Galois group. We consider a Galois cocycle $a\left(\sigma_{1}, \sigma_{2}, \ldots, \sigma_{n}\right)\left(\sigma_{1}, \sigma_{2}, \ldots, \sigma_{n}\right.$ $E G$ ) taking values in the multiplicative group $K^{*}$ of $K$;

Received August 30, 1952.

1: If the 2-cohomology group in $K / k$ vanishes, so do all (1-, 2- and) $3-, 4-, \ldots, n$-cohomology groups; see Hochschild-Nakayama [2], \$4.

2: Observe, for example, that the subgroup of $K^{*} N_{K / k}^{k}$ consisting of all elements left invariant under $G$ is in general greater than $k^{*} N_{k / K}^{*}$

2) See a correction at the end of the present note. 


$$
\begin{aligned}
(\delta a)\left(\sigma_{1}, \sigma_{2}, \ldots, \sigma_{n+1}\right)=a\left(\sigma_{2}, \sigma_{3}, \ldots, \sigma_{n+1}\right) a\left(\sigma_{1} \sigma_{2}, \sigma_{3}, \ldots, \sigma_{n+1}\right)^{-1} \\
\ldots a\left(\sigma_{1}, \sigma_{2}, \ldots, \sigma_{n} \sigma_{n+1}\right)^{(-1)^{n}} a\left(\sigma_{1}, \sigma_{2}, \ldots, \sigma_{n}\right)^{(-1)^{n+1} \sigma_{n+1}}=1 .
\end{aligned}
$$

For an $i=2,3, \ldots, n$ we let $\sigma_{i}$ run over $G$ in (1), to obtain ${ }^{4)}$

$$
\begin{aligned}
& a\left(\sigma_{2}, \ldots, \sigma_{i-1}, G, \sigma_{i+1}, \ldots, \sigma_{n+1}\right) a\left(\sigma_{1} \sigma_{2}, \ldots, \sigma_{i-1}, G, \sigma_{i+1}, \ldots\right)^{-1} \\
& \quad \ldots a\left(\sigma_{1}, \ldots, \sigma_{i-2}, G, \sigma_{i+1}, \ldots\right)^{i-1)^{i-1}} a\left(\sigma_{1}, \ldots, \sigma_{i-1}, G, \sigma_{i+2}, \ldots\right)^{i-1)^{i}} \\
& \quad \ldots a\left(\sigma_{1}, \ldots, \sigma_{i-1}, G, \sigma_{i+1}, \ldots, \sigma_{n}\right)^{(-1)^{n+1} \sigma_{n+1}=1}
\end{aligned}
$$

where $a\left(\sigma_{2}, \ldots, \sigma_{i-1}, G, \sigma_{i+1}, \ldots, \sigma_{n+1}\right)$, for instance, denotes the product $\Pi_{o \in G} a\left(\sigma_{1}, \ldots, \sigma_{i-1}, \sigma, \sigma_{i+1}, \ldots, \sigma_{n+1}\right)$. This means that the product of the value at $\sigma_{1}, \ldots, \sigma_{i-1}$ of the coboundary of the $(i-2)$-cochain $a\left(\sigma_{1}, \ldots, \sigma_{i-2}\right.$, $\left.G, \sigma_{i+1}, \ldots, \sigma_{n+1}\right)$ with respect to $\sigma_{1}, \ldots, \sigma_{i-2}$ in the non-Galois sense $(G$ operating trivially on $K^{*}$ ) (with parameter values $\left.\sigma_{i+1}, \ldots, \sigma_{n+1}\right)$ and $(-1)^{i}$ times the value at $\sigma_{i+1}, \ldots, \sigma_{n+1}$ of the coboundary of the $(n-i)$-cochain $a\left(\sigma_{1}\right.$, $\left.\ldots, \sigma_{i-1}, G, \sigma_{i+1}, \ldots, \sigma_{n}\right)$ with respect to $\sigma_{i+1}, \ldots, \sigma_{n}$ in the usual Galois sense (with parameter values $\sigma_{1}, \ldots, \sigma_{i-1}$ ) is equal to 1 . (As a matter of fact, this fact, with varying $i$, can best be formulated in terms of a double cochain, whose $(i-1, n-i)$-component is $a\left(\sigma_{1}, \ldots, \sigma_{i-1}, G, \sigma_{i+1}, \ldots, \sigma_{n}\right)$; cf. [1] for instance.) For $i=2$ our relation shows that $a\left(\sigma_{1}, G, \sigma_{3}, \ldots, \sigma_{n}\right)$, with a fixed $\sigma_{1}$, is an $(n-2)$-cocycle on $\sigma_{3}, \ldots, \sigma_{n}$. It is easy to see that if the original $n$-cocycle $a\left(\sigma_{1}, \sigma_{2}, \ldots, \sigma_{n}\right)$ is $\sim 1$ then our $a\left(\sigma_{1}, G, \sigma_{3}, \ldots, \sigma_{n}\right)$ is $\sim 1$, for every $\sigma_{1}$. For $i=3$ our relation shows that $a\left(\sigma_{2}, G, \sigma_{1}, \ldots, \sigma_{n+1}\right) a\left(\sigma_{1} \sigma_{2}\right.$, $\left.G, \sigma_{4}, \ldots, \sigma_{n+1}\right)^{-1} a\left(\sigma_{1}, G, \sigma_{4}, \ldots, \sigma_{n+1}\right)$, as an $(n-2)$-cocycle with respect to the last $n-2$ arguments, is $\sim 1$, or, the cohomology class of our $(n-2)$-cocycle $a\left(\sigma_{1}, G, \sigma_{3}, \ldots, \sigma_{n}\right)$ depends multiplicatively on the parameter $\sigma_{1}$.

2. 1- and 2-cocycles in the norm class group associated with an n-cocycle. Let again $a\left(\sigma_{1}, \sigma_{2}, \ldots, \sigma_{n}\right)$ be a (Galois) $n$-cocycle in the Galois extension $K / k$, with Galois group $G$. Set $n=2 m$ or $n=2 m+1$ according as $n$ is even or odd. By the construction of the preceding section we get an $(n-2)$-cocycle $a\left(\sigma_{1}, G\right.$, $\sigma_{3}, \ldots, \sigma_{n}$ ) for each $\sigma_{1}$ (provided $n \geqslant 3$ ). Applying the same construction to this $(n-2)$-cocycle, we get further an $(n-4)$-cocycle, depending on two parameters (provided $n \geqslant 5$ ). Thus, after applying the same construction $m-1$ times, we arrive at a 2 - or 3-cocycle, which depends on $m-1$ parameters, according as $n$ is even or odd. Then we apply either the construction in [3] or the one in [5] to this (2- or 3-) cocycle according as $n=2 m$ or $2 m+1$, to obtain either a 1-cocycle or a 2-cocycle in the norm class group $k^{*} / N_{K / k}^{*}$ (where $N_{K / l}^{*}$ denotes the to ality of norms of the non-zero elements of $K$ with respect to $K / k$ ). Explicitly it is given by $a\left(\sigma_{1}, G, \sigma_{3}, G, \ldots, G, \sigma_{n-1}, G\right)$ in case $n=2 m$, where $\sigma_{1}$, $\sigma_{3}, \ldots, \sigma_{n-3}$ are parameters and $\sigma_{n-1}$ is the cocycle variable. Since this de-

4) On the other hand, if we let $\sigma_{n+1}$ run over $G$ in (1), we are led to a non-Galois $(n-1)$ cocycle modulo $N_{K / k}^{*}$ (which underlies our passage to the norm class group), while the relation obtained by letting $\sigma_{1}$ run is rather well-known. 
pends also multiplicatively on each of the parameters $\sigma_{1}, \ldots, \sigma_{n-3}$, we have

THEOREM 1. If $n=2 m$ we have

$$
a\left(\sigma_{1}, G, \sigma_{3}, G, \ldots, \sigma_{n-1}, G\right) \in k^{*}
$$

for every $\sigma_{1}, \sigma_{3}, \ldots, \sigma_{n-1}$, and for each $i=0,1, \ldots, m$ the mapping $\sigma \rightarrow a\left(\sigma_{1}\right.$, $\left.G, \ldots, \sigma_{2 i-1}, G, \sigma, G, \sigma_{2 i+3}, \ldots, G\right)$ is a homomorphism of $G$ into the norm class group $k^{*} / N_{K / k}^{*}$, which is determined uniquely by the cohomology class of $a(\ldots)$.

As for $n=2 m+1$ our 3 -cocycle is $a\left(\sigma_{1}, G, \sigma_{3}, \ldots, G, \sigma_{n-2}, \sigma_{n-1}, \sigma_{n}\right)$, where $\sigma_{1}, \sigma_{3}, \ldots, \sigma_{n-1}$ are parameters, and to this we may apply the construction in [5]. It was to form the 1-cocycle $a\left(\sigma_{1}, G, \sigma_{2}, \ldots, G, \sigma_{n-2}, G, \sigma_{n}\right)$ on $\sigma_{n}$, to set

$$
a\left(\sigma_{1}, G, \sigma_{3}, \ldots, G, \sigma_{n-2}, G, \sigma_{n}\right)=c\left(\sigma_{1}, \sigma_{3}, \ldots, \sigma_{n-2}\right)^{1-\sigma_{n}}
$$

by virtue of the Hilbert-Speiser theorem, and to form $a\left(\sigma_{1}, G, \sigma_{3}, G, \ldots, G\right.$, $\left.\sigma_{n-2}, \sigma_{n-1}, G\right) c\left(\sigma_{1}, \sigma_{3}, \ldots, \sigma_{n-4}, \sigma_{n-2}\right)^{-1} c\left(\ldots, \sigma_{n-2} \sigma_{n-1}\right) c\left(\ldots, \sigma_{n-1}\right)^{-1}\left(\in k^{*}\right)$. But here we want to make an a little more general construction, which contains this as a special case. Thus, let $\sigma_{2}, \ldots, \sigma_{2 i}, \sigma_{2 i+3}, \sigma_{2 i+5}, \ldots, \sigma_{n}\left(=\sigma_{2 m+1}\right)$ run, independently, over $G$ in (1), for each $i=0,1, \ldots, m-1$, to obtain $a\left(G, \sigma_{3}\right.$, $\left.G, \ldots, \sigma_{2 i-1}, G, \sigma_{2 i+1}, \sigma_{2 i+2}, G, \sigma_{2 i+4}, \ldots, G, \sigma_{n+1}\right) a\left(G, \sigma_{3}, G, \ldots, \sigma_{2 i-1}, G, \sigma_{2 i+1}\right.$, $\left.\sigma_{2 i+2}, G, \sigma_{2 i+4}, \ldots, G, \sigma_{n+1}\right)^{-1} \ldots a\left(\sigma_{1}, G, \sigma_{3}, \ldots, \sigma_{2 i-1}, G, \sigma_{2 i+2}, G, \sigma_{2 i+4}, \ldots, G\right.$, $\left.\sigma_{n+1}\right) a\left(\sigma_{1}, G, \sigma_{3}, \ldots, \sigma_{2 i-1}, G, \sigma_{2 i+1} \sigma_{2 i+2}, G, \sigma_{2 i+4}, \ldots, G, \ldots\right)^{-1} a\left(\sigma_{1}, G, \sigma_{3}, \ldots\right.$, $\left.\sigma_{₫ i-1}, G, \sigma_{2 i+1}, G, \sigma_{2 i+4}, \ldots\right) \ldots a\left(\sigma_{1}, G, \sigma_{3}, \ldots, G, G, \sigma_{n+1}\right)^{-1} a\left(\sigma_{1}, G, \ldots, G\right.$, $\left.G, \sigma_{n+1}\right) a\left(\sigma_{1}, G, \sigma_{3}, G, \ldots, \sigma_{2 i-1}, G, \sigma_{2 i+1}, \sigma_{2 i+2}, G, \sigma_{2 i+4}, \ldots, \sigma_{n-1}, G\right)^{-1} a\left(\sigma_{1}, G\right.$, $\left.\sigma_{3}, G, \ldots, \sigma_{2 i-1}, G, \sigma_{2 i+1}, \sigma_{2 i+2}, G, \sigma_{2 i+4}, \ldots, \sigma_{n-1}, G\right)^{\sigma_{n+1}}=1$, that is

$$
a\left(\sigma_{1}, G, \sigma_{3}, \ldots, \sigma_{2 i-1}, G, \sigma_{2 i+1}, \ldots, G, \sigma_{n+1}\right) \boldsymbol{a}\left(\ldots, \sigma_{2 i-1}, G\right. \text {, }
$$
$\left.\sigma_{2 i+1} \sigma_{2 i+2}, \ldots\right)^{-1} a\left(\ldots, \sigma_{2 i-1}, G, \sigma_{2 i+2}, \ldots\right)=a\left(\sigma_{1}, G, \sigma_{3}, G, \ldots\right.$, $\left.\sigma_{2 i-1}, G, \sigma_{2 i+1}, \sigma_{2 i+2}, G, \sigma_{2 i+1}, \ldots, \sigma_{n-1}, G\right)^{1-\sigma_{n+1}}$.

In view of (4) we put

$$
\begin{aligned}
& \alpha^{(i)}\left(\sigma_{1}, \sigma_{3}, \ldots, \sigma_{2 i-1} ; \sigma_{2 i+1}, \sigma_{2 i+2} ; \sigma_{2 i+1}, \ldots, \sigma_{n-1}\right)=a\left(\sigma_{1}, G, \sigma_{3},\right. \\
& \left.\quad G, \ldots, \sigma_{2 i-1}, G, \sigma_{2 i+1}, \sigma_{2 i+2}, G, \sigma_{2 i+4}, \ldots, \sigma_{n-1}, G\right) c\left(\sigma_{1}, \sigma_{3}, \ldots,\right. \\
& \left.\quad \sigma_{2 i-1}, \sigma_{2 i+1}, \sigma_{2 i+1}, \ldots, \sigma_{n-1}\right)^{-1} c\left(\ldots, \sigma_{2 i-1}, \sigma_{2 i+1} \sigma_{2 i+3}, \sigma_{2 i+4}, \ldots\right) \\
& \quad c\left(\ldots, \sigma_{2 i-1}, \sigma_{2 i+2}, \sigma_{2 i+4}, \ldots\right)^{-1},
\end{aligned}
$$

and find

$$
\alpha^{(i)}\left(\sigma_{1}, \sigma_{3}, \ldots, \sigma_{2 i-1} ; \sigma_{2 i+1}, \sigma_{2 i+2} ; \sigma_{2 i+4}, \ldots, \sigma_{n-1}\right) \in k^{*} .
$$

Further, letting $\sigma_{2}, \sigma_{4}, \ldots, \sigma_{2 i}, \sigma_{2 i+4}, \sigma_{2 i+6}, \ldots, \sigma_{n-1}, \sigma_{n+1}$ run over $G$ in (1), we get, after cancellation,

$$
\begin{aligned}
& a\left(\sigma_{1}, G, \sigma_{3}, \ldots, \sigma_{2 i-1}, G, \sigma_{2 i+2}, \sigma_{2 i+3}, G, \sigma_{2 i+5}, \ldots, \sigma_{n}, G\right) a\left(\ldots, \sigma_{2 i-1},\right. \\
& \left.\quad G, \sigma_{2 i+1} \sigma_{2 i+2}, \sigma_{2 i+3}, G, \ldots\right)^{-1} a\left(\ldots, \sigma_{2 i-1}, G, \sigma_{2 i+1}, \sigma_{2 i+2} \sigma_{2 i+3},\right. \\
& G, \ldots) a\left(\ldots, \sigma_{2 i-1}, G, \sigma_{2 i+1}, \sigma_{2 i+2}, G, \ldots\right)^{-1} N a\left(\sigma_{1}, G, \sigma_{3}, \ldots,\right. \\
& \left.\quad G, \sigma_{2 i+1}, \sigma_{2 i+2}, \sigma_{2 i+3}, G, \ldots, G, \sigma_{n}\right)=1 .
\end{aligned}
$$


In view of the form (6) of $\alpha^{(i)}$ we see that

$$
\begin{aligned}
& \alpha^{(i)}\left(\sigma_{1}, \sigma_{3}, \ldots, \sigma_{2 i-1} ; \sigma, \tau ; \sigma_{2 i+1}, \ldots, \sigma_{n-1}\right) \alpha^{(i)}(\ldots ; \rho \sigma, \tau ; \ldots)^{-1} \\
& \alpha^{(i)}(\ldots ; \rho, \sigma \tau ; \ldots) \alpha^{(i)}(\ldots ; \rho, \sigma ; \ldots)^{-1} \in N_{K / k}^{*} .
\end{aligned}
$$

Thus we have obtained a 2 -cocycle $\alpha^{(i)}(\ldots ; \sigma, \tau ; \ldots)$ in $k^{*}$ modulo $N_{K / k}^{*}$, depending on the parameters $\sigma_{1}, \sigma_{3}, \ldots, \sigma_{2 i-1}$ and $\sigma_{2 i+1}, \ldots, \sigma_{n-1}$ in $G$. We want to see that the cohomology class of this 2-cocycle is determined uniquely by the cohomology class of $a\left(\sigma_{1}, \ldots, \sigma_{n}\right)$. For that purpose, we first observe that $c\left(\sigma_{1}, \sigma_{3}, \ldots, \sigma_{n-2}\right)$ in (4) is determined up to a factor $\gamma\left(\sigma_{1}, \sigma_{3}, \ldots, \sigma_{n-2}\right)$ in $k^{*}$. Then the form (6) of $\alpha^{(i)}$ shows that this factor $\gamma\left(\sigma_{1}, \ldots, \sigma_{n-2}\right)$ gives to it a factor $r(\ldots, \sigma, \ldots)^{-1} \gamma(\ldots, \sigma \tau, \ldots) \gamma(\ldots, \tau, \ldots)^{-1}$ which is a coboundary (in $\left.k^{*}\right)$. Thus the cohomology class of $\alpha^{(i)}\left(\bmod N_{K / k}^{*}\right)$ is determined uniquely by $a(\ldots)$, and the dependency is clearly multiplicative. Now, if $a\left(\sigma_{1}\right.$, $\left.\ldots, \sigma_{n}\right)=(\delta b)\left(\sigma_{1}, \ldots, \sigma_{n}\right)$ with some $(n-1)$-cochain $b\left(\sigma_{1}, \ldots, \sigma_{n-1}\right)$, then

$$
a\left(\sigma_{1}, G, \sigma_{3}, G, \ldots, G, \sigma_{n}\right)=b\left(\sigma_{1}, G, \sigma_{3}, G, \ldots, G\right)^{1-\sigma_{n}}
$$

as we calculate readily. So we may put, in this case, $c\left(\sigma_{1}, \sigma_{3}, \ldots, \sigma_{n-2}\right)=b\left(\sigma_{1}\right.$, $\left.G, \sigma_{3}, \ldots, \sigma_{n-2}, G\right)$. Then $\alpha^{(i)}\left(\sigma_{1}, \ldots ; \sigma, \tau ; \sigma_{2 i+1}, \ldots\right)=a\left(\sigma_{1}, G, \ldots, G, \sigma\right.$, $\tau, G, \ldots) b\left(\sigma_{1}, G, \ldots, G, \sigma, G, \ldots\right)^{-1} b\left(\sigma_{1}, G, \ldots, G, \sigma \tau, G, \ldots\right) b\left(\sigma_{1}, G, \ldots\right.$, $G, \tau, G, \ldots)^{-1}=b\left(G, \sigma_{3}, G, \ldots, G, \sigma, \tau, G, \ldots\right) b\left(G, \sigma_{3}, G, \ldots, G, \sigma, \tau\right.$, $G, \ldots)^{-1} \ldots b\left(\sigma_{1}, G, \sigma_{3}, \ldots, G, \tau, G, \ldots\right) b\left(\sigma_{1}, G, \ldots, G, \sigma \tau, G, \ldots\right)^{-1} b\left(\sigma_{1}\right.$, $G, \ldots, G, \sigma, G, \ldots) \ldots N b\left(\sigma_{1}, G, \sigma_{3}, \ldots, G, \sigma, \tau, G, \ldots, G, \sigma_{n-1}\right) b\left(\sigma_{1}, G, \ldots\right.$ $G, \sigma, G, \ldots)^{-1} b\left(\sigma_{1}, G, \ldots, G, \sigma \tau, G, \ldots\right) b\left(\sigma_{1}, G, \ldots, G, \tau, G, \ldots\right)^{-1}=N b\left(\sigma_{1}\right.$, $G, \ldots, G, \sigma, \tau, G, \ldots) \in N_{K / k}^{*}$. Thus the cohomology class of $\alpha^{(i)}$ depends only on the cohomology class of $a(\ldots)$, in fact multiplicatively.

Next we show that the cohomology class of $\alpha^{(i)}$ depends multiplicatively on each of the parameters $\sigma_{1}, \sigma_{3}, \ldots, \sigma_{2 i-1}, \sigma_{2 i+4}, \ldots, \sigma_{n-1}$. To do so, we let in (1) $\sigma_{2}, \sigma_{4}, \ldots, \sigma_{2 i}, \sigma_{2 i+3}, \sigma_{2 i+5}, \ldots, \sigma_{2 j+1}, \sigma_{2 j+1}, \sigma_{2 j+6}, \ldots, \sigma_{n+1}$ run over $G$ (where $j>i$ ), and obtain, after cancellation,

$$
\begin{gathered}
a\left(\sigma_{1}, G, \sigma_{3}, \ldots, G, \sigma_{2 i+2}, G, \ldots, G, \sigma_{2 j+2}, \sigma_{2 j+3}, G, \ldots\right) a(\ldots, G, \\
\left.\sigma_{2 i+1} \sigma_{2 i+2}, G, \ldots\right)^{-1} a\left(\ldots, G, \sigma_{2 i+1}, G, \ldots\right) a\left(\sigma_{1}, G, \sigma_{3}, \ldots, G,\right. \\
\left.\sigma_{2 i+1}, \sigma_{2 i+2}, G, \ldots, G, \sigma_{2 j+3}, G, \ldots\right)^{-1} a\left(\ldots, \ldots, G, \sigma_{2 j+2} \sigma_{2 j+3}\right. \\
G, \ldots) a\left(\ldots, G, \sigma_{2 j+2}, G, \ldots\right)^{-1} N a\left(\sigma_{1}, G, \sigma_{3}, \ldots\right)=1 .
\end{gathered}
$$

On the other hand, letting $\sigma_{2}, \sigma_{4}, \ldots, \sigma_{2 i}, \sigma_{2 i+2}, \ldots, \sigma_{2 j}, \sigma_{2 j+3}, \sigma_{2 j+5}, \ldots, \sigma_{n}$ run over $G$ in (1), we obtain, again after cancellation, $a\left(\sigma_{1}, G, \sigma_{3}, \ldots, G, \sigma_{2 j+z}\right.$, $G, \ldots) a\left(\ldots, G, \sigma_{2 j+1}, \sigma_{2 j+2}, G, \ldots\right)^{-1} a\left(\ldots, G, \sigma_{2 j+1}, G, \ldots\right) a\left(\sigma_{1}, G, \sigma_{3}, \ldots\right.$, $\left.G, \sigma_{2 j+1}, \sigma_{2 j+2}, G, \ldots\right)^{-1+o_{n+1}}=1$. This shows that

$$
\begin{aligned}
& \varepsilon\left(\sigma_{1}, \sigma_{3}, \ldots, \sigma_{2 j+1}, \sigma_{2 j+2}, \sigma_{2 j+4}, \ldots\right)=a\left(\sigma_{1}, G, \sigma_{3}, \ldots, G, \sigma_{2 j+1},\right. \\
& \left.\sigma_{2 j+2}, G, \ldots\right) c\left(\sigma_{1}, \sigma_{3}, \ldots, \sigma_{2 j-1}, \sigma_{2 j+1}, \sigma_{2 j+4}, \ldots\right)^{-1} c(\ldots, \\
& \left.\sigma_{2 j+1} \sigma_{2 j+2}, \ldots\right) c\left(\ldots, \sigma_{2 j+2}, \ldots\right)^{-1} \in k^{*} .
\end{aligned}
$$


On combining (11) and (12) we have

$$
\begin{aligned}
& \alpha^{(i)}\left(\ldots ; \sigma, \tau ; \ldots \sigma_{2 j+1}, \ldots\right) \alpha^{(i)}(\ldots ; \sigma, \tau ; \ldots, \ldots, \\
& \left.\sigma_{2 j+1} \sigma_{2 j-2} \ldots\right)^{-1} \alpha^{(i)}\left(\ldots ; \sigma, \tau ; \ldots, \sigma_{2 j+2}, \ldots\right)=\varepsilon(\ldots, \sigma, \ldots, \\
& \left.\sigma_{2 j+1}, \sigma_{2 j+2}, \ldots\right) \varepsilon\left(\ldots, \sigma \tau, \ldots, \sigma_{2 j+1}, \sigma_{2 j+2}, \ldots\right)^{-1} \varepsilon(\ldots, \\
& \left.\tau, \ldots, \sigma_{2 j+1}, \sigma_{2 j+2}, \ldots\right) N a\left(\sigma_{1}, G, \ldots\right)^{-1} .
\end{aligned}
$$

Thus $\alpha^{(i)}\left(\ldots, \sigma, \tau ; \ldots, \sigma_{2 j+1} \sigma_{2 j+2}, \ldots\right) \sim \alpha^{(i)}\left(\ldots ; \sigma, \tau ; \ldots, \sigma_{2 j+1}\right.$, $\ldots) \alpha^{(i)}\left(\ldots ; \sigma, \div ; \ldots, \sigma_{2 j+2}, \ldots\right) \bmod N_{K / k}^{*}\left(\right.$ in $\left.k^{*}\right)$. Similarly we see that the cohomology class of $\alpha^{(i)}\left(\bmod N_{K / k}^{*}\right)$ depends multiplicatively on any one of the first $i$ parameters $\sigma_{1}, \sigma_{3}, \ldots, \sigma_{2_{2}-i}$, and we have

Theorem 2. Let $n=2 m+1$ be odd. For every Galois $n$-cocycle $a\left(\sigma_{1}, \ldots\right.$, $\left.\sigma_{n}\right)$ in $K k$ and $i=0,1, \ldots m-1, \alpha^{(i)}\left(\sigma_{1}, \sigma_{3}, \ldots, \sigma_{2 i-1} ; \sigma, \tau ; \sigma_{2 i+1}, \ldots, \sigma_{n-1}\right)$ defined by (6) (where $c\left(\sigma_{1}, \sigma_{3}, \ldots \sigma_{n-2}\right)$ is defined by (4)) gives a 2-cocycle in the norm class group $k^{*}, N_{K / k}^{*}$ (in the ground field). Its cohomology class is determined uniquely by the cohomology class of $a\left(\sigma_{1}, \ldots, \sigma_{n}\right)$, depends multiplicatively on the latter, and depends multiplacatively on each of the parameters $\sigma_{1}, \ldots \sigma_{2 i-1}, \sigma_{2 i-1}, \ldots, \sigma_{n-1}$.

Next we shall see briefly that the vanishing of certain cohomology classes (other than automatically vanishing 1-dimensional ones) enables us to obtain cocycles in the norm class group $k^{*}, N_{K / k}^{*}$ higher than 2-dimensional, as has been indicated in [5] (cf. Baer [1] too). Namely, assume that each $(n-2)$ cocycle $a\left(\sigma_{1}, G, \sigma_{3}, \sigma_{1}, \ldots, \sigma_{n}\right)$ on $\sigma_{3}, \sigma_{4}, \ldots, \sigma_{n}$ (with parameter $\sigma_{1}$ ) is $\sim 1$, i.e.

$$
a\left(\sigma_{1}, G, \sigma_{3}, \sigma_{4}, \ldots, \sigma_{n}\right)=\left(\delta c^{\sigma_{1} j}\right)\left(\sigma_{3}, \ldots, \sigma_{n}\right)
$$

with an $(n-3)$-cochain $c^{\cdot \sigma_{1}}\left(\sigma_{2}, \ldots, \sigma_{n-1}\right)$ (depending on $\left.\sigma_{1}\right)$. Then we construct

$$
a\left(\sigma_{1}, \sigma_{2}, G, \sigma_{4}, \ldots, \sigma_{n}\right) /\left(\partial c\left(\sigma_{4}, \ldots, \sigma_{n}\right)\right)^{\left\{\sigma_{1}, \sigma_{2}\right)}
$$

where $\partial$ is the non-Galois cobundary operation with respect to the parametrical argument $\sigma_{1}$ (whence $\left(\partial c\left(\sigma_{4}, \ldots, \sigma_{n}\right)\right)^{\sigma_{1}, \sigma_{2}}=c^{\left(\tau_{2}\right)}\left(\sigma_{4}, \ldots, \sigma_{n}\right) c^{\left(\gamma_{1}, \sigma_{2}^{i}\right.}\left(\sigma_{4}, \ldots\right.$, $\left.\left.\sigma_{n}\right)^{-1} c^{\left(\gamma_{1}\right)}\left(\sigma_{1}, \ldots, \sigma_{n}\right)\right)$. On account of the case $i=3$ of $(2)$ we find that this $(15)$ is an $(n-3)$-cocyle, on $\sigma_{1}, \ldots, \sigma_{n}$. Assume that this $(n-3)$-cocycle is $\sim 1$, i.e. is equal to the coboundary $\left(\delta d^{1 \sigma_{1}, \sigma_{2}}\right)\left(\sigma_{1}, \ldots, \sigma_{n}\right)$ of an $(n-4)$-cocycle $d^{\left.1 \sigma_{1}, \sigma_{2}\right)}\left(\sigma_{1}, \ldots\right.$, $\left.\sigma_{n-1}\right)$. Then we form $a\left(\sigma_{1}, \sigma_{2}, \sigma_{3}, G, \sigma_{5}, \ldots \sigma_{n}\right)\left(\partial d\left(\sigma_{5}, \ldots, \sigma_{n}\right)\right)^{\left(\sigma_{1}, \sigma_{2}, \sigma_{3},\right.}$, and find, by virtue of the case $i=4$ of $(2)$, that this is an $(n-4)$-cocycle on $\sigma_{5}$, $\ldots, \sigma_{n}$. We assume that this is $\sim 1$, and proceed similarly. Thus, on assuming that certain successively appearing $(n-2)-,(n-3)-, \ldots, 2$-cocycles

$$
\begin{gathered}
a\left(\sigma_{1}, G, \sigma_{3}, \ldots, \sigma_{n}\right), a\left(\sigma_{1}, \sigma_{2}, G, \sigma_{1}, \ldots, \sigma_{n}\right) /\left(\partial c\left(\sigma_{1}, \ldots, \sigma_{n}\right)\right)^{\left(\sigma_{1}, \sigma_{2}\right)}, \\
\ldots, a\left(\sigma_{1}, \ldots, \sigma_{n-2,}, G, \sigma_{n-1}, \sigma_{n}\right)\left(\partial e\left(\sigma_{n-1}, \sigma_{n}\right)\right)^{\left(\sigma_{1}, \sigma_{2}, \ldots, \sigma_{n-3}\right)(-1)^{n-2}}
\end{gathered}
$$

to be $\sim 1$, we arrive at a 1 -cocycle 


$$
\left.a\left(\sigma_{1}, \sigma_{2}, \ldots, \sigma_{n-2}, G, \sigma_{n}\right)\left(\partial f\left(\sigma_{n}\right)\right)^{\left(\sigma_{1}, \sigma_{2}, \ldots, \sigma_{n-2}\right)(-1)^{n-1}} \text { (on } \sigma_{n}\right) .
$$

Then, this is equal to $\left(g^{\left(\sigma_{1}, \sigma_{2}, \ldots, \sigma_{n-2}\right)}\right)^{1-\sigma_{n}}$ with certain $g^{\left(\sigma_{1}, \ldots, \sigma_{n-2}\right)}$. By the case $i=n-1$ of (2) we have that the 0 -cochain $a\left(\sigma_{1}, \ldots, \sigma_{n-1}, G\right)(\partial g)^{\left(\sigma_{1}, \ldots, \sigma_{n-1}\right)(-1)^{n}}$ is a cocycle, i.e.

$$
a\left(\sigma_{1}, \ldots, \sigma_{n-1}, G\right)(\partial \mathrm{g})^{\left(\sigma_{1}, \ldots, \sigma_{n-1}\right)(-1)^{n}} \in k^{*} .
$$

Here $a\left(\sigma_{1}, \ldots, \sigma_{n-1}, G\right)$ is a non-Galois cocycle, in $K^{*}, \bmod N_{K / k}^{*}$ with respect to $\sigma_{1}, \ldots, \sigma_{n-1}$, as we see by letting $\sigma_{n+1}$ run over $G$ in (1). So, putting the left-hand side of (17) $\beta\left(\sigma_{1}, \sigma_{2}, \ldots, \sigma_{n-1}\right)$, we see that $\beta\left(\sigma_{1}, \sigma_{2}, \ldots, \sigma_{n-1}\right)$ is an $(n-1)$-cocycle in the norm class group $k^{*} / N_{k / k}^{*}$. The cohomology class of $\beta$ depends only on the cohomology class of our original cocycle $a$, and in fact multiplicatively. Indeed, if $a \sim 1$ and is $=\delta b$, then we may choose for $c^{\left(\sigma_{1}\right)}\left(\sigma_{3}\right.$, $\left.\ldots, \sigma_{n-1}\right), d^{\left(\sigma_{1}, \sigma_{2}\right)}\left(\sigma_{1}, \ldots, \sigma_{n-1}\right), \ldots, f^{\left(\sigma_{1}, \ldots, \sigma_{n-3}\right)}\left(\sigma_{n-1}\right), g^{\left(\sigma_{1}, \ldots, \sigma_{n-2}\right)}$ successively $b\left(\sigma_{1}, G, \sigma_{3}, \ldots, \sigma_{n-1}\right), b\left(\sigma_{1}, \sigma_{2}, G, \sigma_{4}, \ldots, \sigma_{n-1}\right), \ldots, b\left(\sigma_{1}, \ldots, \sigma_{n-3}, G\right.$, $\left.\sigma_{n-1}\right)^{\left(-1, \rho^{n-2}\right.}, b\left(\sigma_{1}, \ldots, \sigma_{n-2}, G\right)^{(-1)^{n-1}}$, and then we have $a\left(\sigma_{1}, \ldots, \sigma_{n-1}, G\right)$ $(\partial g)^{\left(\sigma_{1}, \ldots, \sigma_{n-1}\right)(-1)^{n}}=b\left(\sigma_{1}, \ldots, \sigma_{n-1}, G\right) b\left(\sigma_{1} \sigma_{2}, \ldots, \sigma_{n-1}, G\right)^{-1} \ldots N b\left(\sigma_{1}, \ldots\right.$, $\left.\sigma_{n-1}\right)^{(-1)^{n}}\left(b\left(\sigma_{2}, \ldots, \sigma_{n-1}, G\right) b\left(\sigma_{1} \sigma_{2}, \ldots, \sigma_{n-1}, G\right) \ldots\right)^{-1}=N b\left(\sigma_{1}, \ldots, \sigma_{n-1}\right)^{-1}$ $\in N_{K / k \text {. }}^{*}$

Remark. Naturaily we may combine these two constructions together.

3. Generalization of the Witt-Akizuki formula. Let now $H$ be a subset of $G$ which is set-wise invariant under every inner automorphism in $G$, and let $h$ be the number of elements in $H$. Letting $\sigma_{1}$ run over $H$ in (1), we have

$$
\begin{aligned}
& a\left(\sigma_{2}, \ldots, \sigma_{n+1}\right)^{h}=\Pi_{i=1}^{n} a\left(H, \sigma_{2}, \ldots, \sigma_{i} \sigma_{i+1}, \ldots, \sigma_{n+1}\right)^{(-1)^{i-1}} a(H, \\
& \left.\sigma_{2}, \ldots, \sigma_{n}\right)^{\left(-1 ; n^{n} \sigma_{n+1}\right.}=\left(\delta a_{1}\right)\left(\sigma_{2}, \ldots, \sigma_{n+1}\right) a\left(H, \sigma_{3}, \ldots, \sigma_{n+1}\right)^{-1} a\left(H \sigma_{2}\right. \text {, } \\
& \left.\sigma_{3}, \ldots, \sigma_{n+1}\right) \text {, }
\end{aligned}
$$

where $a\left(H, \sigma_{2}, \ldots, \sigma_{i} \sigma_{i+1}, \ldots, \sigma_{n+1}\right)$, for instance, denotes $\Pi_{\sigma \in H} a\left(\sigma, \sigma_{2}, \ldots\right.$, $\left.\sigma_{i} \sigma_{i+1}, \ldots, \sigma_{n+1}\right)$ and $a_{1}$ is the $(n-1)$-cochain $a_{1}\left(\sigma_{2}, \ldots, \sigma_{n}\right)=a\left(H, \sigma_{2}, \ldots\right.$, $\left.\sigma_{n}\right)$. Thus

$$
a\left(\sigma_{2}, \ldots, \sigma_{n+1}\right)^{h} \sim a\left(H, \sigma_{2}, \ldots, \sigma_{n+1}\right)^{-1} a\left(H \sigma_{2}, \sigma_{3}, \ldots, \sigma_{n+1}\right) .
$$

Letting $\sigma_{2}$ run over $H$ in (1) we have

$$
\begin{aligned}
& a\left(H, \sigma_{3}, \ldots, \sigma_{n+1}\right) a\left(\sigma_{1} H, \sigma_{3}, \ldots, \sigma_{n+1}\right)^{-1} a\left(\sigma_{1}, H \sigma_{3}, \sigma_{1}, \ldots,\right. \\
& \left.\quad \sigma_{n+1}\right) \prod_{i=3}^{n} a\left(\sigma_{1}, H, \sigma_{3}, \ldots, \sigma_{i} \sigma_{i+1}, \ldots, \sigma_{n+1}\right)^{(-1)^{i}} a\left(\sigma_{1}, H, \ldots,\right. \\
& \left.\sigma_{n}\right)^{(-1)^{u+1} \sigma_{n+1}}=1 .
\end{aligned}
$$

Replacing here $\sigma_{1}$ by $\sigma_{2}$ (and observing that $H$ is self-conjugate in $G$ ) we see that the right-hand side of (19) is equal to

$$
\begin{gathered}
a\left(\sigma_{2}, H \sigma_{3}, \ldots, \sigma_{n+1}\right) \Pi_{3}^{n} a\left(\sigma_{2}, H, \sigma_{3}, \ldots, \sigma_{i} \sigma_{i+1}, \ldots, \sigma_{n+1}\right)^{(-1)^{2}} a\left(\sigma_{2},\right. \\
\left.H, \ldots, \sigma_{n}\right)^{(-1)^{n+1} \sigma_{n+1}}=\left(\delta a_{2}\right)\left(\sigma_{2}, \ldots, \sigma_{n+1}\right)^{-1} a\left(\sigma_{3}, H, \sigma_{4}, \ldots,\right. \\
\left.\sigma_{n+1}\right) a\left(\sigma_{2} \sigma_{3}, H, \sigma_{4}, \ldots, \sigma_{n+1}\right)^{-1} a\left(\sigma_{2}, H \sigma_{3}, \ldots, \sigma_{n+1}\right)
\end{gathered}
$$


where $a_{2}\left(\sigma_{2}, \sigma_{3}, \ldots, \sigma_{n}\right)=a\left(\sigma_{2}, H, \sigma_{3}, \ldots, \sigma_{n}\right)$. Thus

$$
\begin{array}{r}
a\left(\sigma_{2}, \ldots, \sigma_{n+1}\right)^{h} \sim a\left(\sigma_{3}, H, \sigma_{4}, \ldots, \sigma_{n+1}\right) a\left(\sigma_{2} \sigma_{3},\right. \\
\left.H, \sigma_{4}, \ldots, \sigma_{n+1}\right)^{-1} a\left(\sigma_{2}, H \sigma_{3}, \ldots, \sigma_{n+1}\right) .
\end{array}
$$

Letting next $\sigma_{3}$ run over $H$ in (1) and putting $a_{3}\left(\sigma_{2}, \ldots, \sigma_{n}\right)=a\left(\sigma_{2}, \sigma_{3}, H\right.$, $\left.\ldots, \sigma_{n}\right)$ we see that the right-hand side of $(22)$ is equal to $\left(\delta a_{3}\right)\left(\sigma_{2}, \ldots\right.$, $\left.\sigma_{n+1}\right) a\left(\sigma_{3}, \sigma_{4}, H, \ldots, \sigma_{n+1}\right)^{-1} a\left(\sigma_{2} \sigma_{3}, \sigma_{4}, H, \sigma_{5}, \ldots, \sigma_{n+1}\right) a\left(\sigma_{2}, \sigma_{3} \sigma_{4}, H, \sigma_{5}, \ldots\right.$, $\left.\sigma_{n+1}\right)^{-1} a\left(\sigma_{2}, \sigma_{3}, H \sigma_{4}, \sigma_{5}, \ldots, \sigma_{n+1}\right)$. And, continuing in this way, we obtain

$$
\begin{gathered}
a\left(\sigma_{2}, \ldots, \sigma_{n+1}\right)^{h} \sim\left(a ( \sigma _ { 3 } , \ldots , \sigma _ { n + 1 } , H ) \Pi _ { 2 } ^ { n } a \left(\sigma_{2}, \ldots, \sigma_{i} \sigma_{i+1}, \ldots, \sigma_{n},\right.\right. \\
\left.H)^{(-1)^{i-1}} a\left(\sigma_{2}, \ldots, \sigma_{n}, H \sigma_{n+1}\right)\right)^{(-1)^{n}} .
\end{gathered}
$$

But this right-hand side is equal to $\Pi_{\sigma \in H} a\left(\sigma_{2}, \ldots, \sigma_{n+1}\right)^{\sigma}$ as we see by letting $\sigma_{n+1}$ run over $H$ in (1) and replacing $\sigma_{1}, \ldots, \sigma_{n}$ by $\sigma_{2}, \ldots, \sigma_{n+1}$. So we get

$$
a\left(\sigma_{2}, \ldots, \sigma_{n+1}\right)^{h} \sim \Pi_{\sigma \in H} a\left(\sigma_{2}, \ldots, \sigma_{n+1}\right)^{\sigma} .
$$

Now we assume that $H$ is an (invariant) subgroup of $G$. Choosing a representative system in $G$ modulo $H$, we denote, for each element $\sigma$ in $G$, the representative of $\sigma$ by $\sigma_{0}$. The right-hand side of (19) is naturally equal to $a\left(H, \sigma_{3}, \ldots, \sigma_{n+1}\right)^{-1} a\left(H\left(\sigma_{2}\right)_{0}, \ldots, \sigma_{n+1}\right)$, which is, by (20) and (21), in turn equal to $\left(\delta a_{2}\right)\left(\left(\sigma_{2}\right)_{0}, \ldots, \sigma_{n+1}\right)^{-1} a\left(\sigma_{3}, H, \sigma_{1}, \ldots, \sigma_{n+1}\right) a\left(\left(\sigma_{2}\right)_{0 \sigma_{3}}, H, \ldots, \sigma_{n+1}\right)^{-1} a\left(\left(\sigma_{2}\right)_{0}\right.$, $\left.H \sigma_{3}, \ldots, \sigma_{n+1}\right)=\left(\delta a_{2}^{0}\right)\left(\sigma_{2}, \ldots, \sigma_{n+1}\right)^{-1} a\left(\left(\sigma_{3}\right)_{0}, H, \sigma_{4}, \ldots, \sigma_{n+1}\right) a\left(\sigma_{3}, H, \sigma_{4}\right.$, $\left.\ldots, \sigma_{n+1}\right)^{-1} a\left(\left(\sigma_{2} \sigma_{3}\right)_{0}, H, \ldots, \sigma_{n+1}\right)^{-1} a\left(\left(\sigma_{2}\right)_{0} \sigma_{3}, H, \ldots, \sigma_{n+1}\right) a\left(\sigma_{3}, H, \sigma_{4}, \ldots \ldots\right.$ $\left.\sigma_{n+1}\right) a\left(\left(\sigma_{2}\right)_{0} \sigma_{3}, H, \ldots, \sigma_{n+1}\right)^{-1} a\left(\left(\sigma_{2}\right)_{0}, H_{\sigma_{3}}, \ldots, \sigma_{n+1}\right)=\left(\delta a_{2}^{0}\right)\left(\sigma_{2}, \ldots, \sigma_{n+1}\right) a\left(\left(\sigma_{3}\right)_{0}\right.$, $\left.H, \sigma_{4}, \ldots, \sigma_{n+1}\right) a\left(\left(\sigma_{2} \sigma_{3}\right)_{0}, H, \ldots, \sigma_{n+1}\right)^{-1} a\left(\left(\sigma_{2}\right)_{0}, H\left(\sigma_{3}\right)_{0}, \ldots, \sigma_{n+1}\right)$, where $\boldsymbol{a}_{2}^{0}\left(\sigma_{2}\right.$, $\left.\ldots, \sigma_{n}\right)=a\left(\left(\sigma_{2}\right)_{0}, H, \sigma_{3}, \ldots, \sigma_{n}\right)$. So

$$
\begin{aligned}
& a\left(\sigma_{2}, \ldots, \sigma_{n+1}\right)^{h} \sim a\left(\left(\sigma_{3}\right)_{0}, H, \sigma_{4}, \ldots, \sigma_{n+1}\right) a\left(\left(\sigma_{2}\right)_{0}\left(\sigma_{3}\right)_{0}, H, \sigma_{1}, \ldots,\right. \\
& \left.\sigma_{n+1}\right)^{-1} a\left(\left(\sigma_{2}\right)_{0}, H\left(\sigma_{3}\right)_{0}, \sigma_{1}, \ldots, \sigma_{n+1}\right)\left(a \left(\left(\sigma_{2}\right)_{0}\left(\sigma_{2}\right)_{0}, H, \ldots,\right.\right. \\
& \left.\left.\sigma_{n+1}\right) a\left(\left(\sigma_{2} \sigma_{3}\right)_{0}, H, \ldots, \sigma_{n+1}\right)^{-1}\right) .
\end{aligned}
$$

The product of the first three factors in the right-hand side is equal to $\left(\delta a_{3}\right)\left(\left(\sigma_{2}\right)_{0}\right.$, $\left.\left(\sigma_{3}\right)_{0}, \sigma_{4}, \ldots, \sigma_{n+1}\right) a\left(\left(\sigma_{3}\right)_{0}, \sigma_{1}, H, \ldots, \sigma_{n+1}\right)^{-1} a\left(\left(\sigma_{2}\right)_{0}\left(\sigma_{3}\right)_{0}, \sigma_{4}, H, \ldots, \sigma_{n+1}\right) a\left(\left(\sigma_{2}\right)_{0}\right.$, $\left.\left(\sigma_{3}\right)_{0 \sigma_{4}}, H, \ldots, \sigma_{n+1}\right)^{-1} a\left(\left(\sigma_{2}\right)_{0},\left(\sigma_{3}\right)_{0}, H \sigma_{4}, \ldots, \sigma_{n+1}\right)=\left(\delta a_{3}^{0}\right)\left(\sigma_{2}, \sigma_{3}, \ldots, \sigma_{n+1}\right) a\left(\left(\sigma_{3}\right)_{0}\right.$, $\left.\left(\sigma_{4}\right)_{0}, H, \ldots, \sigma_{n+1}\right)^{-1} a\left(\left(\sigma_{2} \sigma_{3}\right)_{0}, \quad\left(\sigma_{4}\right)_{0}, H, \ldots, \sigma_{n+1}\right) a\left(\left(\sigma_{2}\right)_{0}, \quad\left(\sigma_{3} \sigma_{1}\right)_{0}, H, \ldots\right.$, $\left.\sigma_{n+1}\right)^{-1} a\left(\left(\sigma_{2}\right)_{0},\left(\sigma_{3}\right)_{0}, H\left(\sigma_{4}\right)_{0}, \ldots, \sigma_{n+1}\right)$, where $a_{3}^{2}\left(\sigma_{2}, \sigma_{3}, \ldots, \sigma_{n}\right)=\boldsymbol{a}\left(\left(\sigma_{2}\right)_{0},\left(\sigma_{3}\right)_{0}\right.$, $\left.H, \ldots, \sigma_{n}\right)$. Thus we have

$$
\begin{aligned}
& a\left(\sigma_{2}, \ldots, \sigma_{n+1}\right)^{h} \sim a\left(\left(\sigma_{3}\right)_{0},\left(\sigma_{4}\right)_{0}, H, \ldots, \sigma_{n+1}\right)^{-1} a\left(\left(\sigma_{2}\right)_{0}\left(\sigma_{3}\right)_{0},\left(\sigma_{4}\right)_{0},\right. \\
& \left.H, \ldots, \sigma_{n+1}\right) a\left(\left(\sigma_{2}\right)_{0},\left(\sigma_{3}\right)_{1}\left(\sigma_{4}\right)_{0}, H, \ldots, \sigma_{n+1}\right)^{-1} a\left(\left(\sigma_{2}\right)_{0},\left(\sigma_{3}\right)_{0},\right. \\
& \left.H\left(\sigma_{4}\right)_{0}, \ldots, \sigma_{n+1}\right)\left(a ( ( \sigma _ { 2 } ) _ { 0 } ( \sigma _ { 3 } ) _ { 0 } , ( \sigma _ { 1 } ) _ { 0 } , H , \ldots , \sigma _ { n + 1 } ) ^ { - 1 } a \left(\left(\sigma_{2} \sigma_{3}\right)_{0},\left(\sigma_{4}\right)_{0},\right.\right. \\
& H, \ldots)\left(a ( ( \sigma _ { 2 } ) _ { 0 } , ( \sigma _ { 3 } ) _ { 0 } ( \sigma _ { 1 } ) _ { 0 } , H , \ldots ) a \left(\left(\sigma_{2}\right)_{0},\left(\sigma_{3} \sigma_{4}\right)_{0},\right.\right. \\
& \left.H, \ldots)^{-1}\right)\left(a\left(\left(\sigma_{2}\right)_{0}\left(\sigma_{3}\right)_{0}, H, \ldots\right) a\left(\left(\sigma_{2} \sigma_{3}\right)_{0}, H, \ldots\right)^{-1}\right) .
\end{aligned}
$$

Continuing in this manner we obtain 


$$
\begin{aligned}
& a\left(\sigma_{2}, \ldots, \sigma_{n+1}\right)^{h} \sim\left(( \delta a _ { n - 1 } ) ( ( \sigma _ { 2 } ) _ { 0 } , \ldots , ( \sigma _ { n + 1 } ) _ { 0 } ) a \left(\left(\sigma_{2}\right)_{0}, \ldots,\left(\sigma_{n}\right)_{0}\right.\right. \\
& \left.H)^{(-1)^{n+1} \sigma_{n+1}} a\left(\left(\sigma_{2}\right)_{0}, \ldots,\left(\sigma_{n}\right)_{0}, H\left(\sigma_{n+1}\right)_{0}\right)^{(-1)^{n}}\right)^{1-1 !^{n}}\left(\Pi _ { j = 1 } ^ { n - 1 } \left(a \left(\left(\sigma_{2}\right)_{0}\left(\sigma_{3}\right)_{0},\right.\right.\right. \\
& \left.\left(\sigma_{4}\right)_{0}, \ldots,\left(\sigma_{j+2}\right)_{0}, H, \sigma_{j+3}, \ldots, \sigma_{n+1}\right) a\left(\left(\sigma_{2} \sigma_{3}\right)_{0},\left(\sigma_{4}\right)_{0}, \ldots,\left(\sigma_{j+2}\right)_{0}\right. \text {, } \\
& \left.\left.H, \sigma_{j+3}, \ldots, \sigma_{n+1}\right)^{-1}\right)\left(a \left(\left(\sigma_{2}\right)_{0},\left(\sigma_{3}\right)_{0}\left(\sigma_{1}\right)_{0}, \ldots,\left(\sigma_{j+2}\right)_{0}, H, \sigma_{j+3}, \ldots\right.\right. \text {, } \\
& \left.\left.\sigma_{n+1}\right)^{-1} a\left(\left(\sigma_{2}\right)_{0},\left(\sigma_{3} \sigma_{i}\right)_{0}, \ldots,\left(\sigma_{j+2}\right)_{0}, H, \sigma_{j+3}, \ldots, \sigma_{n+1}\right)\right) \ldots\left(a \left(\left(\sigma_{2}\right)_{0},\right.\right. \\
& \left.\left(\sigma_{3}\right)_{0}, \ldots,\left(\sigma_{j+1}\right)_{0}\left(\sigma_{j+2}\right)_{0}, H, \sigma_{j+3}, \ldots, \sigma_{n+1}\right)^{(-1)^{j-1}} a\left(\left(\sigma_{2}\right)_{0},\left(\sigma_{3}\right)_{0}, \ldots\right. \\
& \left.\left.\left(\sigma_{j+1} \sigma_{j+2}\right)_{0}, H, \sigma_{j+3}, \ldots, \sigma_{n+1}\right)^{(-1) j}\right)^{(-1)^{n}} \text {. }
\end{aligned}
$$

Here the product outside of $\Pi$ is equal to $\Pi_{n \in H} a\left(\left(\sigma_{2}\right)_{0}, \ldots,\left(\sigma_{n+1}\right)_{0}\right)^{3}$, as follows from (1) (with $\left(\sigma_{2}\right)_{0}, \ldots,\left(\sigma_{n+1}\right)_{0}$ in place of $\left.\sigma_{1}, \ldots, \sigma_{n}\right)$. Writing now $\sigma_{1}, \ldots, \sigma_{n}$ instead of $\sigma_{2}, \ldots, \sigma_{n+1}$, we get finally.

Theorem 3. Let $H$ be an invariant subgroup of order $h$ in $G$, and $L$ be the subfield of $K$ belonging to $H$. Then

$$
\begin{aligned}
& a\left(\sigma_{1}, \ldots, \sigma_{n}\right)^{h} \sim N_{K / L} a\left(\left(\sigma_{1}\right)_{0} \ldots \ldots,\left(\sigma_{n}\right)_{0}\right)\left(\Pi _ { j = 1 } ^ { n - 1 } \left(a \left(\left(\sigma_{1}\right)_{0}\left(\sigma_{2}\right)_{0},\left(\sigma_{3}\right)_{0},\right.\right.\right. \\
& \left.\ldots \ldots\left(\sigma_{j-1}\right)_{n}, H, \sigma_{j+2}, \ldots, \sigma_{n}\right) a\left(\left(\sigma_{2} \sigma_{3}\right)_{0},\left(\sigma_{1}\right)_{0}, \ldots,\left(\sigma_{j+1}\right)_{0}, H, \sigma_{j+2},\right. \\
& \left.\left.\ldots \sigma_{n}\right)^{-1}\right)\left(a \left(\left(\sigma_{1}\right)_{0},\left(\sigma_{2}\right)_{0}\left(\sigma_{3}\right)_{0}, \ldots,\left(\sigma_{j+1}\right)_{0}, H, \sigma_{j+2}, \ldots,\right.\right. \\
& \left.\left.\sigma_{n}\right)^{-1} a\left(\left(\sigma_{1}\right)_{0},\left(\sigma_{0} \sigma_{3}\right)_{0} \ldots \ldots\left(\sigma_{j+1}\right)_{n}, H, \sigma_{j+2}, \ldots, \sigma_{n}\right)\right) \ldots\left(a\left(\sigma_{1}\right)_{0},\right. \\
& \left.\left(\sigma_{2}\right)_{0}, \ldots,\left(\sigma_{j}\right)_{0}\left(\sigma_{j-1}\right)_{0}, H . \sigma_{j+2}, \ldots, \sigma_{n}\right)^{(-1))_{j-1}} a\left(\left(\sigma_{1}\right)_{0},\left(\sigma_{2}\right)_{0}, \ldots,\right. \\
& \left.\left.\left(\sigma_{j} \sigma_{j+1}\right)_{0}, H, \sigma_{j+2}, \ldots, \sigma_{n}\right)^{(-1) j}\right)^{-1)^{\prime \prime}},
\end{aligned}
$$

where $\sigma_{0}$ denotes the representative of $\sigma$ in a certain representative system in $G$ modulo $H$.

Though this generalizes the Witt-Akizuki formula for 2-cocyles, we note that the right-hand side depends (not only on the classes of $\sigma_{3}, \ldots, \sigma_{n} \bmod H$ but) on $\sigma_{3}, \ldots \sigma_{n}$ themselves; in this respect there was a falsy argument in $[5]$.

\section{REFERENCES}

[1] R. Baer: The cohomology theory of a pair of groups, Proc. Internat. Congress of Mathematicians, 1950, Vol. II, p. 15.

[2] G. Hochschild-T. Nakayama : Zohomology in class field theory, Ann. Math. 55 (1952), p. 348.

[3] T. Nakayama: Über die Beziehung $z$ wischen den Faktorensystemen und der Normklassengruppe eines galoisschen Erweiterungskourpers, Math. Ann. 112 (1935), p. 85.

[4] T. Nakayama: A theorem on the norm group of a finite extension field, Jap, J. Math. 18 (1943) p. 877.

[5] T. Nakayama: Note on 3-factor sets, Kodai Math. Rep. 3 (1949), p. 11.

Mathematical Institute,

Nagoya University 